\title{
Gonioscopy-A Primer
}

\author{
Syed Shoeb Ahmad \\ Queen Elizabeth Hospital, Kota Kinabalu, Malaysia
}

DOI: https://doi.org/10.17925/USOR.2017.10.01.42

\begin{abstract}
A ssessment of the anterior chamber angle (ACA) is an indispensible investigation for evaluation of glaucoma. The most commonly performed method for the determination of the ACA is gonioscopy. This technique, while being simple, is often hampered by the subjective nature of the procedure, especially in inexperienced hands. This review is intended to improve the knowledge, attitude, and practice among the practitioners regarding the procedure of gonioscopy.
\end{abstract}

\section{Keywords}

Gonioscopy, anterior chamber, glaucoma angle-closure, glaucoma open-angle

Disclosure: Syed Shoeb Ahmad has nothing to disclose in relation to this article. This study involves a review of the literature and did not involve any studies with human or animal subjects performed by any of the authors. No funding was received for the publication of this article.

Acknowledgements: Syed Shoeb Ahmad wishes to thank the Secretariat of the Greek Glaucoma Society, Dr Anton Hommer and the Austrian Glaucoma Society, as well as Dr Khoo Say Peng from the Dr Kong Specialist Eye Centre, Kota Kinabalu, Malaysia for the images and Ms Emmeline Stanislaus for editing the manuscript.

Authorship: All named authors meet the International Committee of Medical Journal Editors (ICMJE) criteria for authorship of this manuscript, take responsibility for the integrity of the work as a whole, and have given final approval to the version to be published.

open Access: This article is published under the Creative Commons Attribution Noncommercial License, which permits any noncommercial use, distribution, adaptation, and reproduction provided the original author(s) and source are given appropriate credit.

Received: December 2, 2017

Accepted: February 7, 2017

Citation: US Ophthalmic Review, 2017;10(1):42-5

Corresponding Author: Syed Shoeb Ahmad, Ophthalmology Department, Queen Elizabeth Hospital, Jalan Kebajikan, 88587, Kota Kinabalu, Malaysia. E: syedshoebahmad@yahoo.com
Gonioscopy is a requisite investigation for all patients with glaucoma. It is a procedure for evaluation of the anterior chamber angle (ACA), utilizing special instruments known as gonio-lenses or -prisms. Alexios Trantas (1867-1961) was the first to use the term "gonioscopy" in 1907 (Figure 1). The term was derived from the Greek word "gonia" meaning angle and "skopein" to observe. Trantas used a direct ophthalmoscope and digital pressure at the limbus to observe the ACA in a patient with keratoglobus. Later, Maxmilian Salzmann (1862-1954) used indirect gonioscopy with a contact lens for examination of the angle (Figure 1). ${ }^{1}$ Therefore, both Trantas and Salzmann are called the "Fathers of gonioscopy". ${ }^{1,2}$

Gonioscopy helps to categorize the type of glaucoma, that is, open- or closed-angle. This directs the ophthalmologist towards the subsequent management of glaucoma. The procedure may also identify any secondary causes of outflow obstruction such as pigment, pseudoexfoliative material, new vessels, angle-recession or foreign bodies in the ACA.

This concise review of gonioscopy is intended for residents and junior doctors who are often intimidated by the complex nature of this procedure. The article describes the optical principles forming the basis of gonioscopy, the types of gonioscopy, the procedure for performing indirect gonioscopy, the difference between direct and indirect gonioscopy, identification of angle structures, grades of angle width, dynamic gonioscopy, flowchart for gonioscopy, and the common errors during performing the procedure.

\section{Principle of gonioscopy}

When light passes from a medium with a greater index of refraction to a medium with lower index of refraction, the angle of refraction ( $r$ ) becomes greater than the angle of incidence (i). i reaches a "critical angle" when $r$ is equal to $90^{\circ}$. If i becomes more than the critical angle, light is reflected back into the first medium (Figure 2).

The critical angle for the cornea-air interface is approximately $40^{\circ}$. Light rays coming from the angle of the anterior chamber exceed the critical angle and are therefore reflected back into the AC. This prevents visualization of the ACA on slitlamp biomicroscopy. This deficiency can be overcome by optically replacing the cornea with another interface. The refractive index of the contact lens is the same as that of the corneal epithelium so that there is minimal refraction at the interface of these two surfaces (contact lens-cornea). This removes the optical effect of cornea. The light rays from the ACA reach the contact lens. Subsequently, they are made to pass through the new contact lens-air interface.

In direct gonioscopy (gonio-lens) the anterior curve of the contact lens is such that the critical angle is not reached. The light rays are refracted at the contact lens-air interface to the observer's eye. In indirect gonioscopy, the light rays are reflected by a mirror in the contact lens (gonioprism). The light rays leave the lens at nearly a right angle to the contact lens-air interface. ${ }^{2}$ 
Figure 1: Alexios Trantas and Maxmillian Salzmann
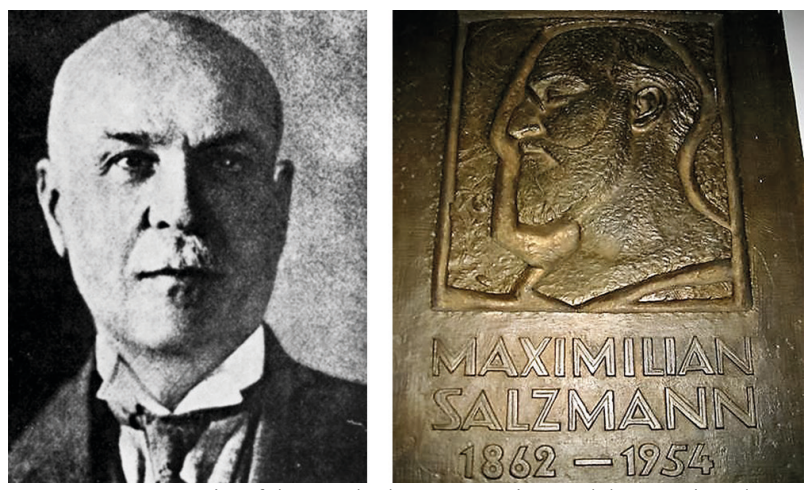

Images courtesy Secretariat of the Greek Glaucoma Society and the Austrian Glaucoma Society.

Figure 2: A diagram showing the angle of incidence $\left(i, i^{\prime}, i^{\prime \prime}\right)$ and the angle of refraction $\left(r, r^{\prime}, r^{\prime \prime}\right)$

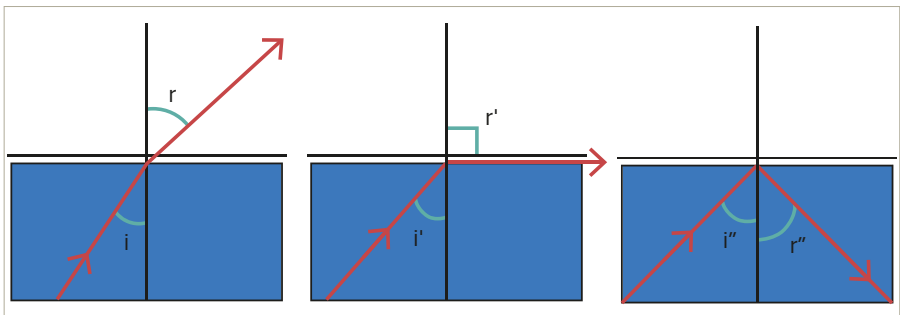

\section{Direct gonioscopy}

Direct gonioscopy can be performed by Koeppe-type lenses. A hand-held slitlamp, gonioscope, or an operating microscope is used along with the goniolenses for evaluation of the angle. This procedure is usually performed on pediatric patients under general anesthesia or sedation. The patient is in a supine position and a coupling fluid is often used to bridge the lens to the cornea, but the patient's own tears can also be used. After inserting the goniolens, the microscope and light source are adjusted to examine the entire angle. Some of these lenses, such as the Barkan, Thorpe, and Swan-Jacob are also used for surgical procedures such as goniotomy.

\section{Indirect gonioscopy}

Indirect gonioscopy utilizes slitlamp biomicroscopy and a contact lens to observe the ACA (Table 1). A comparison of indirect and direct gonioscopy is given in Table 2.

\section{Procedure of indirect gonioscopy}

- Have minimal room illumination.

- With a Goldmann 3-mirror lens, a coupling agent such as GenTeal Gel (Novartis Ophthalmics, Basel, switzerland) (Carbomer 0.22\% and Hypromellose 0.3\%) has to be used. The dome shaped lens is used for gonioscopy. However, with a Zeiss lens, no coupling agent is required.

- Use the shortest slit beam possible (around $2 \mathrm{~mm}$ wide). Do not throw light into the pupil.

- Use high magnification.

- Keep beam off-center at 30-35․

- Place lens gently on the eye with patient looking up. Once the lens is in place ask the patient to look straight ahead.

- Stop moving the lens when you can view the iris (furrows).
Table 1: Types of lenses commonly used

\begin{tabular}{|l|l|l|}
\hline Type of goniolens & Mirror height & Mirror tilt \\
\hline $\begin{array}{l}\text { Goldmann single-mirror contact lens } \\
\text { (Haag-Streit, Köniz, Switzerland) }\end{array}$ & $12 \mathrm{~mm}$ & $62^{\circ}$ \\
\hline $\begin{array}{l}\text { Goldmann 3-mirror (Haag-Streit, Köniz, } \\
\text { Switzerland) }\end{array}$ & & $59^{\circ}$ \\
\hline $\begin{array}{l}\text { Zeiss 4-mirror (Carl Zeiss AG, } \\
\text { Oberkochen, Germany) }\end{array}$ & $64^{\circ}$ \\
\hline $\begin{array}{l}\text { Ritch trabeculoplasty 4-mirror lens } \\
\text { (Ocular Instruments, Bellevue, USA) }\end{array}$ & $\begin{array}{l}2 \text { tilted }=59^{\circ} \\
2 \text { tilted }=62^{\circ}\end{array}$ \\
\hline
\end{tabular}

Table 2: A comparison of direct and indirect gonioscopy

\begin{tabular}{|c|c|}
\hline Indirect & Direct \\
\hline $\begin{array}{l}\text { Advantages } \\
\text { - } \text { Convenient } \\
\text { - Patient need not lie flat } \\
\text { - Slit lamp examination provides } \\
\text { better details compared to direct } \\
\text { gonioscopy techniques } \\
\text { - } \text { Requires less instrumentation } \\
\text { - Less time-consuming } \\
\text { - } \text { Dynamic gonioscopy is possible }\end{array}$ & $\begin{array}{l}\text { Advantages } \\
\text { - Provides a straight-on view. The angle } \\
\text { of visualization can be changed by } \\
\text { altering the height of the observer } \\
\text { which may enable evaluation over } \\
\text { the curvature of iris e.g. iris bombe or } \\
\text { narrow angles } \\
\text { - Less distortion of the AC is produced } \\
\text { by goniolenses } \\
\text { - The view is more panoramic } \\
\text { - Lenses in both eyes simultaneously } \\
\text { can make comparison easier } \\
\text { - Fundus examination through a small } \\
\text { pupil is also possible }\end{array}$ \\
\hline $\begin{array}{l}\text { Disadvantages } \\
\text { - Mirror image is produced } \\
\text { - Inadvertent pressure can open or } \\
\text { close the angle } \\
\text { - Depth of a narrow angle cannot be } \\
\text { seen }\end{array}$ & $\begin{array}{l}\text { Disadvantages } \\
\text { - Inconvenient procedure, with the } \\
\text { patient having to lie supine }\end{array}$ \\
\hline
\end{tabular}

AC $=$ anterior chamber. Modified from: Alward and Longmuir, 2008. ${ }^{3}$

- Each quadrant of the angle is visualized with the opposite mirror.

- Usually, the inferior angle is the deepest and most pigmented. However, in cases of acute angle closure the pigment could be denser superiorly due to the increased apposition of the iris against the trabecular meshwork.

\section{Identification of the angle structures Ciliary body}

Once the iris is visualized, move the beam towards the periphery. In myopes, aphakia/pseudophakia the ciliary body is visible as a pink, dull brown or slate grey band (Figure 3). The width of the ciliary body band depends on the level of iris insertion and is wider in myopes. In case the ciliary body band is abnormally deep and not symmetric with the other eye, one should rule out conditions such as angle recession, cyclodialysis and unilateral high myopia.

\section{Scleral spur}

The scleral spur is the posterior lip of the scleral sulcus. It appears as a prominent white line between the dark ciliary body band and the pigmented trabecular meshwork. Fine, pigmented strands frequently cross the scleral spur. These run from the iris root to the pigmented trabecular meshwork, and are called "iris processes". The scleral spur 
Figure 3: Open angle

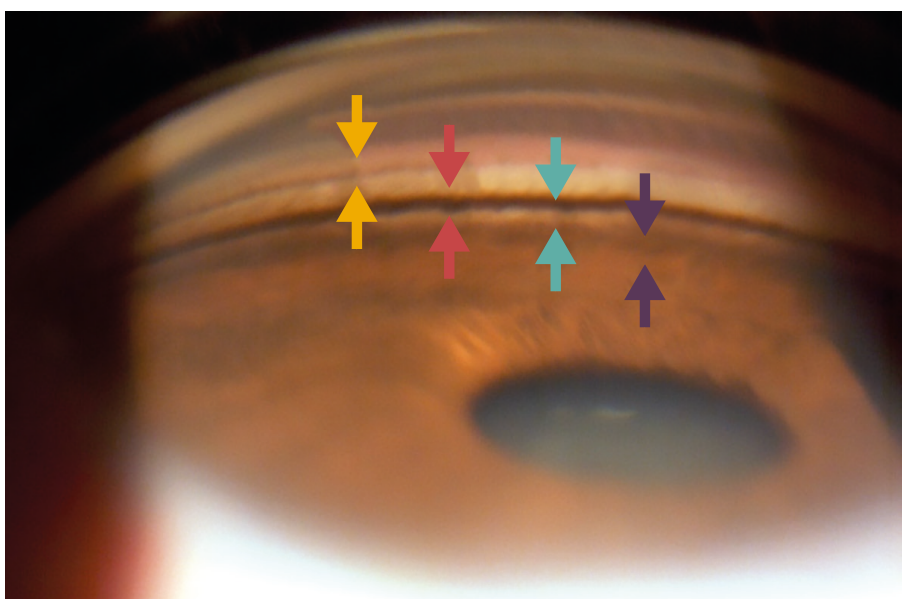

An open angle showing the ciliary body band (between the purple arrows), the scleral spur (between the blue arrows), the functional trabecular meshwork (between the red arrows) and Schwalbe's line (between the yellow arrows).

Figure 4: A closed angle showing a pigmented Schwalbe's line; while other structures are obscured

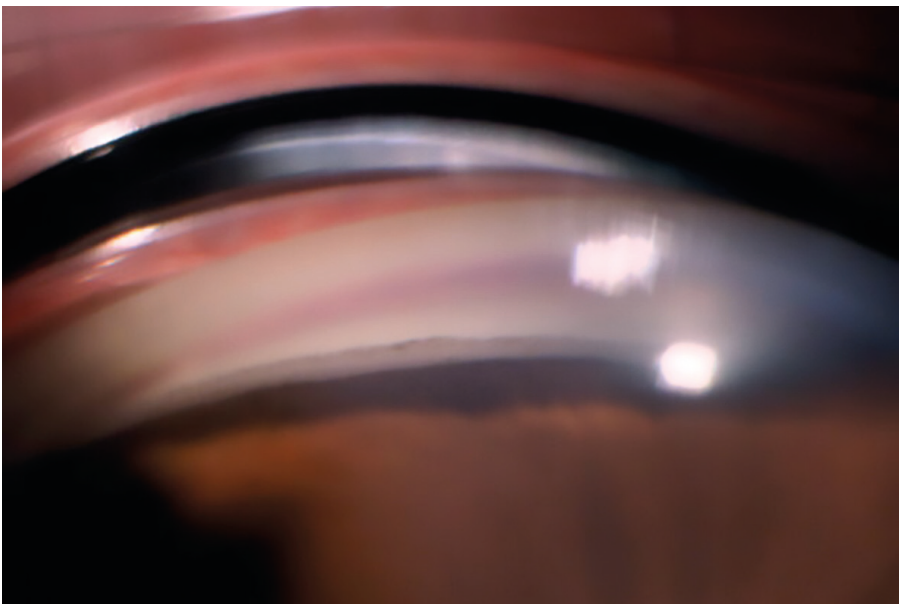

Picture courtesy Dr Khoo Say Peng, Kota Kinabalu, Malaysia.

may also be obscured by high iris insertion, iris bombe, peripheral anterior synechiae (PAS), and due to heavy pigmentation.

\section{Trabecular meshwork}

The trabecular meshwork is approximately $600 \mu$ wide. It has two parts: the anterior non-functional part, which lies adjacent to Schwalbe's line and has a whitish color; and the posterior functional part, which is seen as a grayish-blue pigmented band, just anterior to the scleral spur. This part of the trabecular meshwork is non-pigmented at birth but with aging a faint tan to dark brown color may develop. The color of the trabecular meshwork depends on the degree of pigmentation (Table 3).

\section{Schwalbe's line}

The junction of the angle structures and cornea is called Schwalbe's line (Figure 4). It is the peripheral termination of Descemet's membrane and appears as a fine ridge or opaque line. In highly pigmented eyes, it can be pigmented, especially inferiorly. A prominent and anterior
Table 3: Main causes of trabecular pigmentation

\begin{tabular}{|l|l|}
\hline Physiological & Pathological \\
\hline - Senility & - Pigment dispersion syndrome \\
- Pseudophakic pigment dispersion & - Pseudoexfoliation \\
- Blunt ocular trauma \\
- Anterior uveitis \\
- Following acute angle closure \\
- Following YAG laser iridotomy \\
- Cataract surgery in uncontrolled diabetes mellitus \\
- Nevus of Ota \\
- Iris melanoma
\end{tabular}

YAG = yttrium aluminium garnet. Modified from Kanski, 2007. ${ }^{4}$

Table 4: Grades of angle width

\begin{tabular}{|c|c|c|c|c|}
\hline $\begin{array}{l}\text { ACA } \\
\text { grade }\end{array}$ & Degree & Structure visible & Remarks & Interpretation \\
\hline Grade 4 & $35^{\circ}-45^{\circ}$ & $\begin{array}{l}\text { Ciliary body } \\
\text { visible }\end{array}$ & $\begin{array}{l}\text { Seen in myopia, } \\
\text { aphakia, } \\
\text { pseudophakia }\end{array}$ & Incapable of closure \\
\hline Grade 3 & $25^{\circ}-35^{\circ}$ & $\begin{array}{l}\text { Scleral spur } \\
\text { visible }\end{array}$ & & Incapable of closure \\
\hline Grade 2 & $20^{\circ}$ & $\begin{array}{l}\text { Trabecular } \\
\text { meshwork visible }\end{array}$ & $\begin{array}{l}\text { Moderately } \\
\text { narrow angle }\end{array}$ & $\begin{array}{l}\text { Angle closure possible } \\
\text { but unlikely }\end{array}$ \\
\hline Grade 1 & $10^{\circ}$ & $\begin{array}{l}\text { Schwalbe's line } \\
\text { visible }\end{array}$ & $\begin{array}{l}\text { Very narrow } \\
\text { angle }\end{array}$ & $\begin{array}{l}\text { Angle closure not } \\
\text { inevitable, but risk is } \\
\text { high }\end{array}$ \\
\hline Slit angle & & $\begin{array}{l}\text { No obvious irido- } \\
\text { corneal contact. } \\
\text { However, no } \\
\text { angle structures } \\
\text { can be identified }\end{array}$ & & $\begin{array}{l}\text { High risk of imminent } \\
\text { closure }\end{array}$ \\
\hline Grade 0 & $0^{\circ}$ & $\begin{array}{l}\text { Iridocorneal } \\
\text { contact present. } \\
\text { Inability to } \\
\text { identify the apex } \\
\text { of corneal wedge }\end{array}$ & Closed angle & $\begin{array}{l}\text { Indentation } \\
\text { gonioscopy is required } \\
\text { to differentiate } \\
\text { appositional from } \\
\text { synechial angle closure }\end{array}$ \\
\hline
\end{tabular}

Modified from: Kanski, 2007. ${ }^{4}$

Schwalbe's line is called a "posterior embryotoxon". It could be seen in normal eyes as a physiological variation or could be associated with Axenfeld-Rieger syndrome.

\section{Schlemm's canal}

In non-pigmented eyes, the Schlemm's canal is visible as a slightly darker or red line deep to the posterior trabecular meshwork. Blood may regurgitate into the schlemm's canal due to excessive pressure from the goniolens on the episcleral veins. However, pathological conditions such as carotid cavernous fistula, Sturge-Weber syndrome, and obstructed superior vena cava may also raise the episcleral venous pressure and lead to blood in schlemm's canal.

\section{Corneal wedge}

This technique can be used in identification of an inconspicuous Schwalbe's line. When a thin slit beam is thrown on the cornea, two shiny lines are seen, one on the external surface and another on the inner surface of 
the cornea. These two lines meet at the apex of the corneal wedge which denotes the Schwalbe's line.

\section{Blood vessels}

Normally, blood vessels run in a radial pattern at the base of the angle (iris). However, pathological new vessels in the angle may occur in neovascular glaucoma, Fuchs uveitic syndrome and chronic anterior uveitis. The ciliary body may also normally show circumferentially running vessels.

\section{Grading of the angle}

Once the practitioner is familiar with the angle structures, that knowledge can be applied to grade the angle. A number of classifications have been introduced in order to correlate the gonioscopic appearance of the ACA with the potential for angle closure. Among the common grading systems are the: Scheie, Shaffer, Spaeth and the Kanski methods (Table 4). Based on the gonioscopic findings, the ACA can be categorized into open- or closedangles. This has been highlighted in Figure 5.

\section{Dynamic gonioscopy (compression or indentation gonioscopy)}

When standard gonioscopy reveals an angle in which the trabecular meshwork is not visible, it indicates a narrow or closed angle. This iridotrabecular contact could be due to fixed causes (PAS) or non-fixed appositional closure. These conditions can be differentiated by performing "dynamic, compression or indentation gonioscopy". ${ }^{5}$

In this technique, pressure is applied on the cornea with a contact lens having a small contact area, such as a Zeiss lens, this pushes aqueous into the ACA opening it up. If PAS (synechial closure) are present, the angle may not open on application of pressure. However, if only iris processes are present or the cornea and angle structures are in close approximation (appositional closure), the angle will open up on applying pressure with the goniolens.

PAS may occasionally be confused with iris processes. The latter are small extensions from the anterior surface of the iris which insert around the scleral spur, obscuring the ciliary body to some extent. They do not inhibit the movement of iris with indentation and do not interfere with aqueous outflow. On the contrary, PAS are broad, irregular and attach the iris stroma to the trabecular meshwork. They bridge the angle recess, obscure the deeper structures and unlike iris processes, normal angle structures may not be visible in between the synechiae. PAS also do not open on indentation gonioscopy.

\section{Errors in gonioscopy}

- In certain instances, gonioscopy is a relative contra-indication. These include: Suspicious or known globe laceration or perforation or recent hyphema (in such situations gonioscopy can be deferred for a few weeks), corneal surface disorders, and epithelial basement membrane dystrophy.

- As the cornea is affected by gonioscopy, investigations such as checking the intra-ocular pressure, performing visual fields and optical coherence tomography should be done prior to gonioscopy.
Figure 5: Gonioscopy flowchart

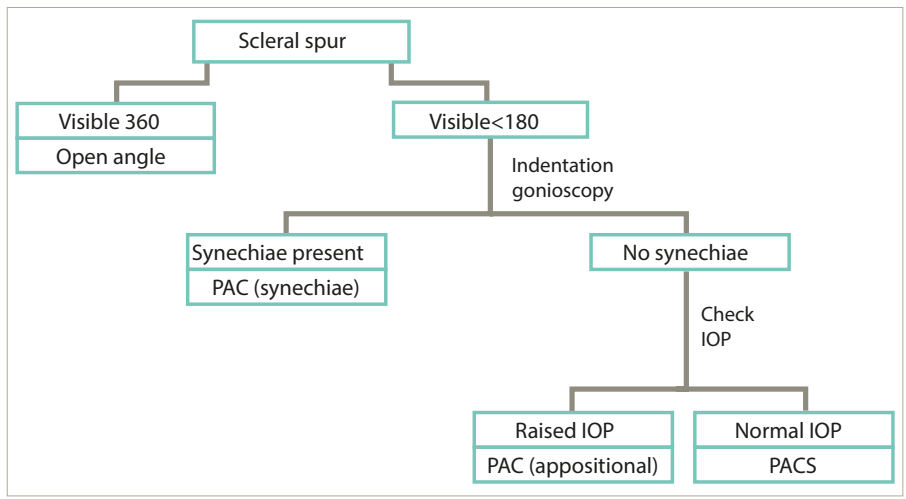

Flowchart for assessment of gonioscopy, based on the guidelines of the Asia Pacific Glaucoma Society, 2016. ${ }^{6} 1 O P=$ intraocular pressure; $P A C=$ primary angle closure; PACS = primary angle closure suspect.

- Bright ambient light can constrict the pupil and falsely open up the angle.

- The three-mirror lenses usually have larger surface area and may be difficult to insert compared to single-mirror or Zeiss lenses in patients with small palpebral apertures.

- Air bubbles under the goniolens may hamper proper visualization of the structures. Tilt the lens towards the bubbles to remove them or take off the lens and re-insert.

- Only half of the goniolens well should be filled up with the coupling fluid.

- Excessive pressure with a Goldmann type lens can compress the eyeball and cause artifactual narrowing of the angle.

- With Zeiss type lenses, excessive pressure opens up the angle and produces corneal striae.

- If the view of the angle is obscured by a convex iris, it is possible to see "over the hill by" asking the patient to look in the direction of the mirror.

- When the plane of the iris is flat, the patient should be asked to look away from the mirror in order to obtain a view parallel to the iris with optimal image quality.

- Pigmentation, on or infront of the Schwalbe's line (Sampaolesi line) might be confused with a pigmented trabecular meshwork and the corneal wedge technique should be used in these instances to confirm the site of Schwalbe's line.

- Prolonged application of the goniolens can cause corneal edema and should be avoided.

\section{Conclusion}

Gonioscopy is a convenient and simple technique which can be done in most patients. However, it is a subjective procedure and needs to be mastered in order to categorize the eye into primary open- or -close angle or secondary glaucoma. Understanding the basic principles pertaining to gonioscopy and remembering the normal appearance of the ACA can help the practitioner to master this vital component in the appraisal of glaucoma. $\square$
1. Faschinger G, Hommer A. Gonioscopy, Berlin, Germany: SpringerVerlag, 2012;1-23.

2. Allingham RR, Damij KF, Freedman $\mathrm{S}$, et al., The basic aspects of glaucoma, gonioscopy and other techniques for assessing the anterior segment. In: Shields Textbook of
Glaucoma 6th ed, Philadelphia, US: Lippincott Williams and Wilkins, 2010;72-80

3. Alward WLM, Longmuir RA, Color atlas of gonioscopy 2nd ed, San Francisco: American Academy of Ophthalmology, 2008

4. Kanski JJ, Clinical Ophthalmology, a systematic approach 6th ed,
Oxford, UK: Elsevier Butterworth Heinemann, 2007:11-15. 5. Weinreb RN, Friedman DS, Angle Closure and Angle Closure Glaucoma Amsterdam, Netherlands: Kugler Publications, 2006. Asia Pacific Glaucoma Guidelines. 3rd ed. Asia pacific Glaucoma Society, 2016. 